\title{
POLARIZATION MEASUREMENTS OF SOME HERBIG Ae/Be STARS
}

\author{
S.K. Jain, H.C.Bhatt, Ram Sagar \\ Indian Institute of Astrophysics \\ Bangalore 560034, India
}

\begin{abstract}
We have measured the linear polarization of 8 bright Herbig $\mathrm{Ae} / \mathrm{Be}$ stars in UBVRI bands. No unique wavelength dependence of polarization magnitude as well as direction is found in these measurements.

As part of a programme of optical polarization studies of star forming regions we have made polarization measurements of eight relatively bright $\mathrm{Ae} / \mathrm{Be}$ stars from the list of Herbig (1960), namely AB Aur, T Ori, V380 Ori, HD 250550, Lk H $\propto$ 215, HD 259431, Z CMa, and HD 53367. The observations were made in March 1989 with the $104 \mathrm{~cm}$ telescope of U.P. State Observatory, Nainital. A fast star-and-sky chopping polarimeter, similar to that described by Viswanathan (1972), was used for the study. The filters are the Fernie combinations of Schott colour glass filters. Each object was observed twice, and all the observations were done with an aperture of 15 arc sec diameter. The degree of polarization, position angle, and their errors were determined by the least-squares method. Several standard stars - both polarized as well as unpolarized - were observed to assess the performance of the instrument. The instrumental polarization is well below $0.1 \%$, and was subtracted from the observations.
\end{abstract}

The results of our measurements are given in table 1. The following points can be made from these observations (i) $A B$ Aur and $T$ Ori have negligible degree of polarization; (ii) Out of the objects with significant

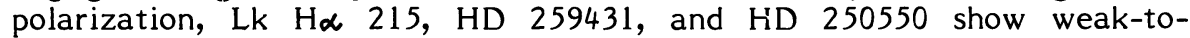
insignificant wavelength dependence of polarization; (iii) Five stars ( Lk H $\alpha$ 215, HD 259431, Z CMa, V380 Ori, and HD 250550) show no wavelength dependence of position angle; (iv) In the other three stars, either the wavelength dependence of position angle is weak (HD 53367 and $\mathrm{AB}$ Aur) or peculiar ( $\mathrm{T}$ Ori).

We plan to continue monitoring these and other Herbig $\mathrm{Ae} / \mathrm{Be}$ stars polarimetrically.

\section{References}

Herbig,G.H. : Ap. J. Suppl. 4, 337 (1960).

Vishwanathan,N. : P.A.S.P. $\underline{84}, 248$ (1972). 
Table 1 : Polarization of the observed Herbig Ae/Be stars

\begin{tabular}{|c|c|c|c|c|c|}
\hline \multirow{2}{*}{$\frac{\text { Object }}{\text { AB Aur }}$} & \multirow{2}{*}{ Filter } & \multicolumn{2}{|c|}{$P \pm \varepsilon(\%)$} & \multicolumn{2}{|c|}{$\theta$ (deg.) } \\
\hline & & 0.05 & \pm 0.08 & 90 & \pm 15 \\
\hline & B & 0.08 & \pm 0.04 & 49 & \pm 14 \\
\hline & V & 0.08 & \pm 0.04 & 91 & \pm 6 \\
\hline & $\mathbf{R}$ & 0.10 & \pm 0.04 & 87 & \pm 6 \\
\hline & I & 0.12 & \pm 0.06 & 92 & \pm 7 \\
\hline \multirow[t]{5}{*}{ T Ori } & $U$ & & - & & - \\
\hline & B & 0.23 & \pm 0.17 & 30 & \pm 24 \\
\hline & V & 0.21 & \pm 0.06 & 68 & \pm 9 \\
\hline & $\mathbf{R}$ & 0.12 & \pm 0.05 & 75 & \pm 10 \\
\hline & I & 0.26 & \pm 0.09 & 25 & \pm 10 \\
\hline \multirow[t]{4}{*}{ V 380 Ori } & $\begin{array}{l}\mathrm{U} \\
\mathrm{B}\end{array}$ & $\begin{array}{l}2.65 \\
1.05\end{array}$ & $\begin{array}{l} \pm 1.53 \\
\pm 0.32\end{array}$ & $\begin{array}{l}139 \\
112\end{array}$ & $\begin{array}{l} \pm 16 \\
\pm 6\end{array}$ \\
\hline & $\mathrm{V}$ & 0.70 & \pm 0.10 & 110 & \pm 4 \\
\hline & $\mathrm{R}$ & 0.55 & \pm 0.06 & 111 & \pm 3 \\
\hline & I & 0.43 & \pm 0.12 & 110 & \pm 7 \\
\hline & U & 1.00 & \pm 0.48 & 110 & \pm 12 \\
\hline \multirow{4}{*}{ (MWC 789) } & B & 1.20 & \pm 0.11 & 171 & \pm 3 \\
\hline & $\mathrm{V}$ & 1.08 & \pm 0.06 & 168 & \pm 1 \\
\hline & $\mathrm{R}$ & 1.00 & \pm 0.05 & 169 & \pm 1 \\
\hline & I & 0.81 & \pm 0.10 & 175 & \pm 4 \\
\hline \multirow[t]{5}{*}{ Lk Ho 215} & $\mathrm{U}$ & & - & & - \\
\hline & B & 1.14 & \pm 0.27 & 81 & \pm 7 \\
\hline & V & 1.10 & \pm 0.08 & 73 & \pm 2 \\
\hline & $\mathrm{R}$ & 1.00 & \pm 0.06 & 80 & \pm 2 \\
\hline & I & 0.99 & \pm 0.10 & 72 & \pm 3 \\
\hline \multirow{5}{*}{$\begin{array}{l}\text { HD } 259431 \\
\text { (NWC 147) }\end{array}$} & $U$ & & - & & - \\
\hline & B & 0.86 & \pm 0.08 & 106 & \pm 3 \\
\hline & V & 0.87 & \pm 0.05 & 106 & \pm 2 \\
\hline & $\mathrm{R}$ & 0.91 & \pm 0.04 & 106 & \pm 1 \\
\hline & I & 0.70 & \pm 0.06 & 105 & \pm 2 \\
\hline Z CMa & U & & - & & - \\
\hline \multirow{4}{*}{ (MWC 165) } & B & 1.51 & \pm 0.34 & 155 & \pm 6 \\
\hline & V & 0.84 & \pm 0.08 & 156 & \pm 6 \\
\hline & $\mathbf{R}$ & 0.79 & \pm 0.04 & 155 & \pm 2 \\
\hline & I & 0.53 & \pm 0.06 & 155 & \pm 3 \\
\hline \multirow{5}{*}{ (MWC 166) } & U & 0.61 & \pm 0.09 & 94 & \pm 4 \\
\hline & B & 0.63 & \pm 0.11 & 55 & \pm 5 \\
\hline & V & 0.30 & \pm 0.05 & 40 & \pm 5 \\
\hline & $\mathbf{R}$ & 0.23 & \pm 0.04 & 67 & \pm 7 \\
\hline & I & 0.16 & \pm 0.06 & 67 & \pm 10 \\
\hline
\end{tabular}

